\title{
Complete Remission of Multicentric Castleman's Disease developed in a HIV+ Patient after the Start of HAART
}

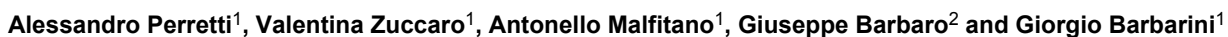 \\ ${ }^{1}$ Infectious Diseases Department, IRCCS Policlinico San Matteo Foundation, University of Pavia, Viale Taramelli, 5 Pavia, Italy \\ ${ }^{2}$ Department of Experimental Medicine, Section, of Medical Pathophysiology, University La Sapienza, Rome, Italy
}

*Corresponding author: Giorgio Barbarini, IRCCS Policlinico San Matteo Foundation, Infectious Diseases Department, University of Pavia, Italy, Tel: 39-368249624; Email: giorgio.barbarini@libero.it

Copyright: ( 2014 Perretti A, et al. This is an open-access article distributed under the terms of the Creative Commons Attribution License, which permits unrestricted use, distribution, and reproduction in any medium, provided the original author and source are credited.

Received date:May 24, 2014; Accepted date: Jul 30, 2014; Published date:August 12, 2014

\begin{abstract}
In August 2010, we diagnosed a Multicentric Castleman's Disease (MCD) in a HIV+ patient with generalized lymphadenopathy, splenomegaly and pericardial effusion. A few days before the onset of symptoms, the patient had started anti-retroviral therapy with Abacavir/Lamivudine (ABC/3TC) +Ritonavir-boosted Atazanavir (ATV/r). During the hospitalization he developed a severe pancytopenia with liver failure, and he started immediately the Therapy with steroids, Ganciclovir, and Rituximab. At the 46 months follow-up, the patient is asymptomatic, his biochemical values are essentially normal with a complete regression of the superficial and deep lymphadenopathies.
\end{abstract}

\section{Key words:}

Castleman disease; Therapy of castleman disease; HHV8; Lymphomas AIDS related Lympho proliferative disorders in HIV

\section{Background}

Castleman's disease (CD) was first described in 1956 by Benjamin Castleman and his colleagues in a group of patients with localized hyperplastic mediastinal lymphadenopathy [1]. CD, also known as angiofollicolar or giant lymph node hyperplasia, is an uncommon lymphoproliferative disorder with heterogeneous manifestations, ranging from asymptomatic disease to recurrent generalised lymphadenopathy with systemic symptoms. The exact incidence of $\mathrm{CD}$ is unknown [2]. Three histologic variants (hyaline vascular, plasma-cellular, and mixed) and two clinical types (localised and multicentric) have been described [3]. Contrary to the benign, localized form, HIV associated multicentric Castleman disease (HIV$\mathrm{MCD}$ ) is a malignant lymphoproliferative disease. It's pathogenesis is unclear, however, HIV-associated MCD, like Kaposi's sarcoma, is strongly associated with Human Herpesvirus 8 (HHV8) [4]. Although HIV-MCD is not classified as a lymphoma or AIDS-defining illness, mortality is high and progression to lymphoma occurs frequently [5]. In recent years, many Authors have pointed out the benefits of treatment with monoclonal anti-CD20 antibodies (Rituximab) associated with highly active antiretroviral therapy (HAART) and Ganciclovir. In a systemic review by Mylona et al. [6], polychemotherapy with $\mathrm{CHOP}$ has given long-term remission in a subset of patients. Other regimens used in the treatment of HIVrelated multicentric Castleman's disease were antiviral agents, immunomodulatory agents, and thalidomide. A retrospective review of 52 patients with HIV-MCD from Germany, (1998 to 2010), compared the use of antiretroviral, antiblastic and Rituximab therapy alone or in combination. The study showed a significant increase in survival in patients treated with Rituximab alone or in combination with other drugs [7]. Rituximab appears efficacious in treating HIV$\mathrm{MCD}$, and its use has been associated with a significant reduction in multiple plasma cytokines [8]. For what concernes the prognosis, a study in the United Kingdom showed that survival at 2 and 5 years after treatment with Rituximab were respectively $94 \%$ and $90 \%$ while these figures were $42 \%$ and $33 \%$ before the introduction of anti-CD20 therapy [9]. Furthermore, a study conducted in 2012 suggests that the recent introduction of rituximab therapy could be associated with long-term protection against the risk of progression to lymphoma [10]. Role of HAART has been also discussed. In 2010, Lee et al. reported the case of 4 patients affected by HIV-MCD successfully treated with antiretroviral therapy alone [11]. Similarly, in 2012 two cases of HIV-associated multicentric Castleman disease treated with HIV antiretroviral therapy were described [12]. In 1999, however, the onset of a rapidly progressive HIV-MCD in subjects who had recently started antiretroviral treatment has been reported [13].

\section{Case presentation}

In April 2010 a 47 year old male patient came to our center for a poll of analysis for sexually transmitted diseases; he diagnosed HIV with HIV RNA 7298 copies/ml and CD4+ T cells 769 cells $/ \mathrm{mm}^{3}$. HBV and HCV markers were negative. In July he started antiretroviral therapy (CD4 398, HIV RNA 38400) with Abacavir/Lamivudine +Ritonavir-boosted Atazanavir. After few weeks later the patient was treated with broad spectrum antibiotics for the onset of intermittent fever, without benefit. At the end of August he was hospitalized to our department for the persistence of fever and chest pain; he also presented laterocervical lymphadenopathy with splenomegaly.

\section{Investigations}

Echocardiographic examination showed mild pericardial effusion. Biochemical investigations were essentially normal, with the exception of a slight increase in the inflammatory markers. Blood and urine cultures, collected at various times without any antibiotic therapyin progress, were negative for bacteria and fungi. A few days after admission the patient showed severe anemia and pancytopenia requiring a transfusion and progressive deterioration of liver function. 
A total body CT scan evidenced lymph nodes enlargement in laterocervical, mediastinal, axillary and sovraclaveare stations, strongly suggestive of lymphoproliferative disease. Therefore, one axillary lymph node was surgically removed for histological diagnosis. Meanwhile, the patient, showed a further deterioration of liver function (prothrombin time 49\%, serum albumin $1.7 \mathrm{~g} / \mathrm{dl}$ ). This induced us to start an empirical treatment with corticosteroids and to switch the antiretroviral therapy to $\mathrm{ABC} / 3 \mathrm{TC}+$ Raltegravir. The histological analysis of the axillary lymph node showed a variable capsular thickening and a B follicular component characterized by hyaline-vascular proliferation, compatible with Castleman's disease. Direct bacteriological examination (including Ziehl-Neelsen staining), as well as bacterial cultures, were negative.

\section{Differential diagnosis}

Patient's symptoms at admission were compatible with immune reconstitution inflammatory syndrome (IRIS). Indeed, the patients have started HAART only four weeks before the onset of symptoms. However, at the start of HAART therapy, his CD4 count was very high, making the diagnosis of IRIS less likely. A disseminated bacterial infection could also be included in the differential diagnosis, but a treatment with broad-spectrum antibiotics was not beneficial.

\section{Treatment}

As for now, no gold standard therapy exists for HIV-MCD. We evaluated several therapeutic possibilities, based on data available in the medical literature, including chemotherapy with Rituximab or Rituximab alone. However, patient's clinical conditions (severe pancytopenia and liver failure) would have not allowed treatment with chemoterapy. After having assessed HHV8 DNA (3109 copies/ml) and IL-6 $(13.01 \mathrm{pg} / \mathrm{ml})$, it was started a therapy with Rituximab $(700 \mathrm{mg} /$ dose) and Ganciclovir. The first dose of Rituximab, probably due to massive release of cytokines resulting from administration of antiCD20, was discontinued due to adverse reaction with shaking chills and sensory impairment. During the following week the patient continued treatment with steroids and Ganciclovir with an improvement in the inflammation indices. Seven days later, the treatment with Rituximab was resumed, in absence of adverse reactions. Subsequent blood tests demonstrated a complete restoring of the liver function and normal cell-blood-count values. After 4 cycles of Rituximab, a control total-body CT scan showed a marked reduction, in volume and number, of the enlarged lymph nodes previously described. Determination for HHV8 DNA in blood was negative. The patient was discharged with only antiretroviral therapy.

\section{Outcome and follow-up}

Viro-immunological follow-ups over a 46 months period showed HIVRNA and HHV8DNA persistently undetectable. CD4+ count were always higher than 650 cells $/ \mathrm{mm}^{3}$. The patient remained completely asymptomatic. Total-body CT scan performed 46 months after the end of therapy with Rituximab, Ganciclovir and steroids showed a complete resolution of lymphadenopathy.

\section{Discussion}

In our opinion, the onset of HIV-MCD after initiation of antiretroviral therapy brings back up the debate on the role of
HAART, not only in therapy, but also in the pathogenesis of this disease. This, however, does not call into question the validity of antiretroviral therapy against HIV-MCD, already documented in the past. Very often the symptoms associated with HIV-MCD can be confused with IRIS, especially if the pathology appears after a few days from starting antiretroviral treatment. For this reason, the histological examination of lymph node biopsies is the most valuable diagnostic element. Our experience adds to that of others, who have successfully used Rituximab for the treatment of HIV-MCD, alone or in combination with other drugs such as steroids or Ganciclovir. From our experience it may be inferred that Rituximab approach may work even when the patient's status is severely compromised providing also a complete remission at 12 month.

\section{Learning points/take home messages}

Our experience confirms the validity of Rituximab and Ganciclovir in the treatment of HIV-MCD. The HIV-MCD must be considered in the differential diagnosis of immune reconstitution inflammatory syndrome, especially when it onsets after starting HAART. The role of HAART in the pathogenesis of HIV-MCD needs further investigation.

\section{References}

1. Castleman B, Iverson L, Menendez VP (1956) Localized mediastinal lymphnode hyperplasia resembling thymoma. Cancer 9: 822-830.

2. Moore DF, Preti A, Tran SM (1996) Prognostic implications following an indeterminate diagnostic work-up of lymphoma. Blood 88: 229a.

3. Keller AR, Hochholzer L, Castleman B (1972) Hyaline-vascular and plasma-cell types of giant lymph node hyperplasia of the mediastinum and other locations. Cancer 29: 670-683.

4. Dupin N, Gorin I, Deleuze J, Agut H, Huraux JM, et al. (1995) Herpeslike DNA sequences, AIDS-related tumors, and Castleman's disease. N Engl J Med 333: 798.

5. Oksenhendler E, Duarte M, Soulier J, Cacoub P, Welker Y, et al. (1996) Multicentric Castleman's disease in HIV infection: a clinical and pathological study of 20 patients. AIDS 10: 61-67.

6. Mylona EE, Baraboutis IG, Lekakis LJ, Georgiou O, Papastamopoulos V, et al. (2008) Multicentric Castleman's disease in HIV infection: a systematic review of the literature. AIDS Rev 10: 25-35.

7. Hoffmann C, Schmid H, Müller M, Teutsch C, van Lunzen J, et al. (2011) Improved outcome with rituximab in patients with HIV-associated multicentric Castleman disease. Blood 118: 3499-3503.

8. Bower M, Veraitch O, Szydlo R, Charles P, Kelleher P, et al. (2009) Cytokine changes during rituximab therapy in $\mathrm{HIV}$-associated multicentric Castleman disease. Blood 113: 4521-4524.

9. Bower M, Newsom-Davis T, Naresh K, Merchant S, Lee B, et al. (2011) Clinical Features and Outcome in HIV-Associated MulticentricCastleman's Disease. J Clin Oncol 29: 2481-2486.

10. Gérard L, Michot JM, Burcheri S, Fieschi C, Longuet P, et al. (2012) Rituximab decreases the risk of lymphoma in patients with HIVassociated multicentric Castleman disease. Blood 119: 2228-2233.

11. Lee SM, Edwards SG, Chilton DN, Ramsay A, Miller RF (2010) Highly active antiretroviral therapy alone may be an effective treatment for HIVassociated multicentric Castleman's disease. Haematologica 95: 1979-1981

12. Rosin C, Hostettler F, Elzi L, Dirnhofer S, Battegay M (2012) Beneficial course of two cases of HIV-associated multicentric Castleman disease treated with HIV antiretroviral therapy. AIDS 26: 1449-1450.

13. Zietz C, Bogner JR, Goebel FD, Löhrs U (1999) An unusual cluster of cases of Castleman's disease during highly active antiretroviral therapy for AIDS. N Engl J Med 340: 1923-1924. 